\title{
FIRST TEST OF ORBIT RESPONSE MATRIX IN PROTON STORAGE RING
}

\author{
C.M. Chu, M. Ball, G. East, B. Hamilton, D. Jeon, X. Kang, L.L. Kiang*, \\ S.Y. Lee, K.Y. $\mathrm{Ng}^{\dagger}$, A. Pei, A. Riabko, T. Sloan, \\ Indiana University Cyclotron Facility \\ 2401 Milo B. Sampson Lane, Bloomington IN 47405
}

\begin{abstract}
The Orbit Response Matrix method is applied to experimentally determine the Cooler ring optics at the Indiana University Cyclotron Facility (IUCF). We have carried out two experiments in Oct. and Nov. 1996 to measure the orbit response of the IUCF Cooler Ring. An analysis software was adopted from the NSLS, BNL[1]. In our analysis, strength error in quadrupoles and steering dipoles, and amplifier gain in BPMs are included as fitting parameters. However, effects of the linear and non-linear coupling are excluded in our preliminary analysis. Since the resolution of our BPM system is of the order of $10 \mu \mathrm{m}$, we will address the effect of BPM resolution on the applicability of the orbit response matrix method in proton storage rings.
\end{abstract}

\section{INTRODUCTION}

Orbit response matrix method has been applied to measure and to calibrate the linear optics in electron storage rings for many years[2, 3, 4, 5, 6]. The method can be used to calibrate the strength and the roll of magnets such as quadrupoles and orbit steerer, or gain factor and roll of beam position monitors (BPMs). In the IUCF Cooler ring, from time to time we encountered difficulty on tuning beam due to unknown device calibration error. Therefore we would like to apply the orbit response matrix method to calibrate the Cooler ring. This would be the first test of orbit response matrix in a proton storage ring, although there is no fundamental difference between an electron ring and a proton ring as far as the method concerning.

The response matrix is defined as

$$
X_{i}=M_{i j} \theta_{j},
$$

where $\mathbf{M}$ is either the measured or model response matrix, $\theta$ is the vector of changes in steering magnet strengths, and $\mathbf{X}$ is the vector of the resulting change in the particle orbit. The basic idea for the response matrix method is to minimize the difference between the measured and model matrices, $\mathbf{M}_{\text {meas }}$ and $\mathbf{M}_{\text {mod }}$, respectively. To perform the minimization we define a vector $\mathbf{V}$ as

$$
V_{k} / \sigma_{l}=\frac{d V_{k} / \sigma_{l}}{d x_{m}} \triangle x_{m}
$$

where $V_{k}=\left|M_{\text {mod }_{i j}}-M_{\text {meas }_{i j}}\right|$ is the difference between the model calculated from either MAD[7] or

\footnotetext{
* also at National Tsing Hua University, Taiwan

${ }^{\dagger}$ Fermilab, P.O. Box 500, Batavia, IL 60510
}

COMFORT[8] program, and the measured orbit response matrices, with $k$ running over all the data points which equals to the multiplication of the number of steering magnets varied and the number of BPMs used; $\sigma_{l}$ 's are measured BPM noise levels which act as weights to different BPMs; $x_{m}$ 's are the parameters varied to minimize the difference between the model and measured response matrix which can be quadrupole strengths, corrector strengths, the BPM gains, or dispersion correction. Note that $\sigma_{k}$ is determined by measuring the orbit many times without changing any corrector strengths. The $\chi^{2}$ to be minimized is given by

$$
\chi^{2}=\sum_{k} \frac{V_{k}^{2}}{\sigma_{l}^{2}} .
$$

By iteratively solving linear Eqs. 2, the $\chi^{2}$ can be minimized and a fitted model can be obtained.

The IUCF Cooler is a proton storage ring with electron cooling. The ring's circumference is about $86.80 \mathrm{~m}$. A unique feature of the Cooler ring is a straight section with electron cooling, which can greatly reduce the proton beam's momentum spread. There are many multiply linked orbit steering magnets in the cooling section in order to maintain the proton beam closed orbit as well as to confine the cooling electron beam in the cooling section. To avoid much complication, we excluded those steering magnets in our fitting program. In our analysis, there are 36 quadrupole magnets, 2 horizontal and 15 vertical steering magnets, and 19 horizontal and 17 vertical BPMs. We varied the steering magnets one by one and recorded all the BPM data. Thus the number of data points is $(19+17) \times(2+15)=612$.

\section{ORBIT RESPONSE EXPERIMENT}

For our analysis, we exclude any linear and nonlinear coupling. A single BPM response time can be adjusted from 20 to $100 \mathrm{~ms}$ depending on the precision requirement. Our experiment was set at $50 \mathrm{~ms}$ for each BPM and a total of $2 \mathrm{~s}$ for BPM data taking in one Cooler cycle. The BPM resolution is about $0.01 \sim 0.04 \mathrm{~mm}$.

Before we started taking orbit response data, the linear betatron coupling in the ring had to be minimized[9, 10], where the beam is kicked in one direction and tuned to minimize the observed motion in the orthogonal plane. This effect can be maximized if the $\mathrm{X}$ and $\mathrm{Y}$ tunes are close to each other. We used a spectrum analyzer to observe the beating oscillations due to the linear betatron coupling fol- 
lowing either a horizontal or a vertical kick. The linear coupling resonance can be corrected by maximizing the beat period of the transverse oscillations using a pair of skew quadrupoles.

However, in this experiment, there was still a nonnegligible linear coupling in the ring. This linear coupling effect would contribute to systematic error. A horizontal closed orbit plot following a vertical kick is shown in Fig.1.

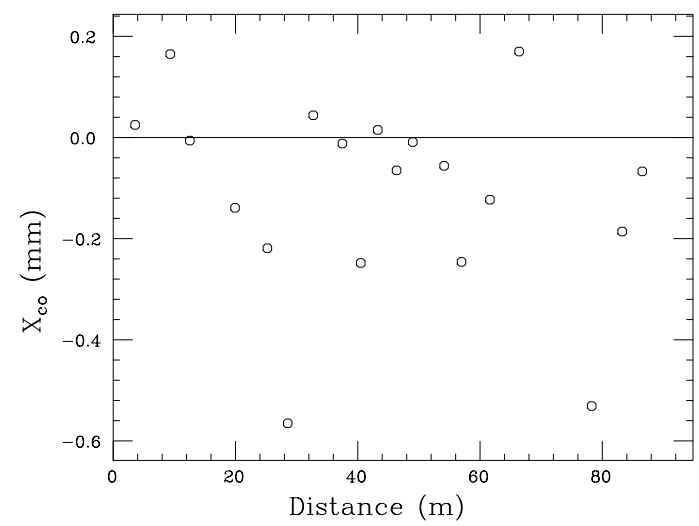

Figure 1: Horizontal closed orbit displacement after a vertical kick after linear coupling minimization. The large nonzero data shows there is still a significant coupling effect.

In the data analysis, the orbit response is assumed to be in a linear range. Thus the next step is to check the linearity of the kicking strength for each kicker versus the orbit response. Fig. 2 shows a typical vertical closed orbit versus steering magnet strength in a high $\beta$ section.

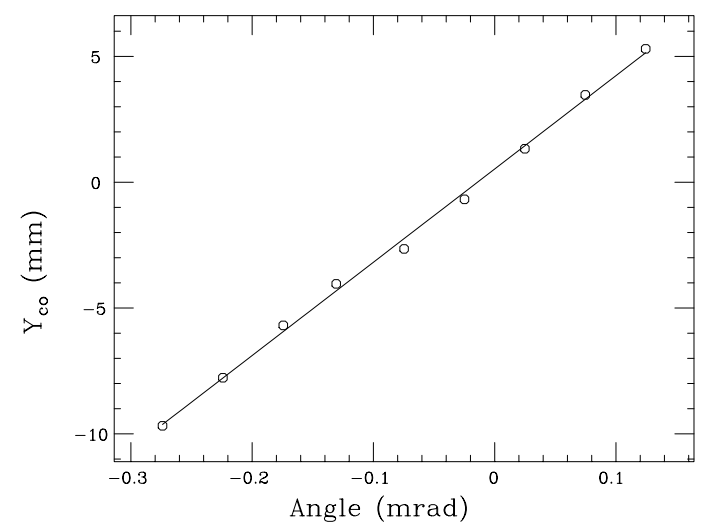

Figure 2: Vertical closed orbit displacement vs. steering magnet strength. The solid line is a fit.

For each run, we set the Cooler cycle time to about $10 \mathrm{~s}$ and took 10 consecutive orbit measurements for each steering magnet strength, and averaged over the 10 readouts to get one BPM closed orbit data. Because of the ring instability, we could only calculate each BPM noise level by averaging over about 10 repeated runs. The noise level of BPMs ranges from 0.03 to $0.1 \mathrm{~mm}$.

\section{DATA ANALYSIS AND RESULTS}

For the parameters to fit, there are 36 quadrupole gradients, 19 horizontal and 17 vertical BPM gains, 2 horizontal and 15 vertical steering magnet strengths, and $2 \Delta p / p$ 's for the 2 horizontal steering magnets. To sum up the above parameters, we then have a total of 91 parameters to fit.

A computer program called $L O C O$ (Linear Optics from Closed Orbit) was adopted from the NSLS, BNL. Originally we tried to fit the quadrupole gradients first. However, because the quadrupole gradients are nonlinear and the number of quadrupole gradients (36) is too large, it is very difficult to find a converged solution in the fitting routine efficiently. Therefore, the fitting strategy we used was to fit only the 17 steering magnet strengths first; after the program converged, added the 19 horizontal BPM gain factors; once it done, then included the 17 vertical BPM gain factors; then added the energy correction for the 2 horizontal steering magnets; and finally included the 36 quadrupole gradients. The $\chi^{2}$ for each step is listed in Table 1 .

Table 1: The Orbit Response fitting results.

\begin{tabular}{||c|c||}
\hline \hline Fit Parameters Included & $\chi^{2}$ \\
\hline 2 horizontal and 15 vertical kickers & $\sim 9500$ \\
\hline $\begin{array}{c}\text { 2 horizontal, 15 vertical kickers } \\
\text { and 19 horizontal BPMs }\end{array}$ & $\sim 9400$ \\
\hline 2 horizontal, 15 vertical kickers, & \\
19 horizontal and 17 vertical BPMs & $\sim 1300$ \\
\hline $\begin{array}{c}2 \text { horizontal, 15 vertical kickers, } \\
19 \text { horizontal, 17 vertical BPMs } \\
\text { and 2 horizontal kicker energy corrections }\end{array}$ & $\sim 1300$ \\
\hline $\begin{array}{c}2 \text { horizontal, 15 vertical kickers, } \\
19 \text { horizontal, 17 vertical BPMs, } \\
2 \text { horizontal kicker energy corrections } \\
\text { and 36 quadrupole gradients }\end{array}$ & $\sim 360$ \\
\hline \hline
\end{tabular}

The best fit to both vertical and horizontal closed orbit for a typical steering magnet is shown in Fig. 3 and Fig. 4, respectively. Although for some steering magnets there are field clamps on and thus quadrupole effect appearing, we were still able to calibrate the vertical steering magnet field strength to about $10 \%$ of accuracy. For the best fit, the vertical closed orbit error is within a standard deviation. However, because there are only 2 horizontal steering magnets included in the analysis, the horizontal fit result is not conclusive.

According to our fit result, we did point out a vertical steering magnet's wrong field strengths by more than $50 \%$. This was proved by measuring the current out of the power supply and the distance between the kicker pole tips.

A difficulty of operating the IUCF Cooler ring is that the beam would not be stored without several sextupole magnets on. This means that we are not able to fit the normal quadrupole gradients without sextupole components. 


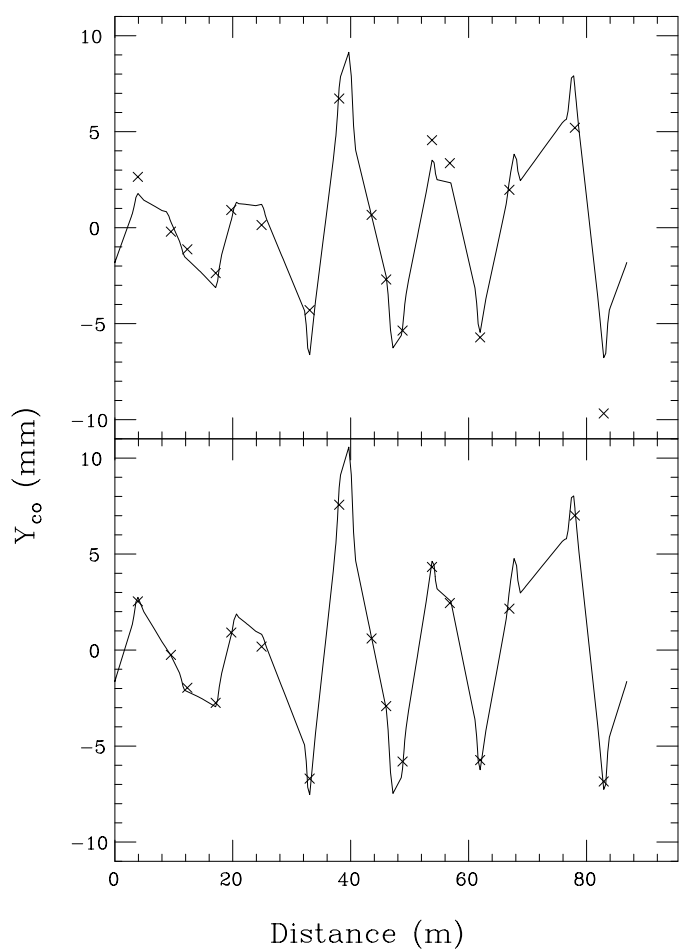

Figure 3: The model and measured vertical closed orbit displacement before (top) and after (bottom) fit. The solid lines are calculated model using COMFORT.

Therefore, there is an unavoidable systematic error due to the sextupole effect.

\section{CONCLUSION}

In general, the orbit response matrix method could be applied to a proton storage ring. At the IUCF Cooler ring, a fundamental problem for the accuracy of the orbit response matrix is that there are only 2 independent steering magnets included in the analysis. Furthermore, due to the insufficiency of the data points, the linear coupling effect can not be taken into account. A direct solution to these problems is to install more horizontal steering magnets.

\section{ACKNOWLEDGMENTS}

We would like to thank James Safranek for the original analysis software and valuable advice. Dick Yoder and Bill Manwaring's help developing the on-line BPM data aquisition was appreciated. We also thank the entire IUCF operation group for their help.

\section{REFERENCES}

[1] J. Safranek, private communication.

[2] J. Safranek and M.J. Lee, "Calibration of the X-Ray Ring Quadrupoles, BPMs, and Orbit Correctors Using the Measured Orbit Response Matrix", Proceedings of Orbit Correction and Analysis in Circular Accelerators Workshop, AIP Conf. Proc. No. 315, 128 (1994).

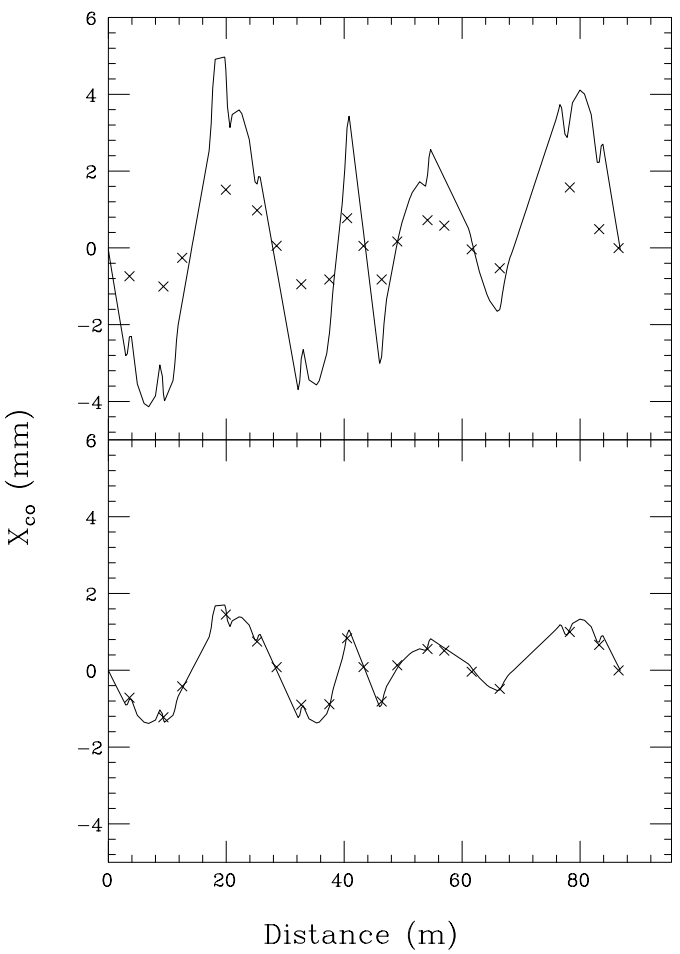

Figure 4: The model and measured horizontal closed orbit displacement before (top) and after (bottom) fit. The solid lines are calculated model using COMFORT.

[3] J. Safranek and M.J. Lee, "Calibration of the X-Ray Ring Quadrupoles, BPMs, and Orbit Correctors Using the Measured Orbit Response Matrix",Proceedings of the $1994 \mathrm{Eu}-$ ropean Particle Accelerator Conference, 1027.

[4] J. Bengtsson and M. Mdedahi, "Modeling of Beam Dynamics and Comparison with Measurements for the Advanced Light Source", Proceedings of the 1994 European Particle Accelerator Conference, 1021.

[5] J. Safranek, "Experimental Determination of Linear Optics Including Quadrupole Rotations", Proceedings of the 1995 IEEE Particle Accelerator Conference, 2817.

[6] D. Robin et al., "Model Calibration and Symmetry Restoration of the Advanced Light Source", Proceedings of the 1996 European Particle Accelerator Conference, to be published.

[7] H. Grote and F.C. Iselin, The MAD Program (Methodical Accelerator Design), Version 8.1, User's Reference Manual, CERN/SL/90-13(AP) (1991).

[8] L. Sanchez et al., COMFORT (Control of Machine Functions, Orbits, and Trajectories) Version 4.0, unpublished.

[9] J.Y. Liu et al., Phys. Rev. E 49, 2347 (1994).

[10] T. Sloan, private communication.

[11] W.H. Press et al., Numerical Recipes in Fortran, The Art of Scientific Computing, 2nd ed., Cambridge University Press (1992). 